\title{
Carbon nanomaterial-derived lung burden analysis using UV-Vis spectrophotometry and proteinase $\mathrm{K}$ digestion
}

\author{
Dong-Keun Lee', Soyeon Jeon ${ }^{1}$, Jiyoung Jeong ${ }^{1}$, Kyung Seuk Song ${ }^{2}$ and Wan-Seob Cho ${ }^{1 *}$ (D
}

\begin{abstract}
Background: The quantification of nanomaterials accumulated in various organs is crucial in studying their toxicity and toxicokinetics. However, some types of nanomaterials, including carbon nanomaterials (CNMs), are difficult to quantify in a biological matrix. Therefore, developing improved methodologies for quantification of CNMs in vital organs is instrumental in their continued modification and application.
\end{abstract}

Results: In this study, carbon black, nanodiamond, multi-walled carbon nanotube, carbon nanofiber, and graphene nanoplatelet were assembled and used as a panel of CNMs. All CNMs showed significant absorbance at $750 \mathrm{~nm}$, while their bio-components showed minimal absorbance at this wavelength. Quantification of CNMs using their absorbance at $750 \mathrm{~nm}$ was shown to have more than 94\% accuracy in all of the studied materials. Incubating proteinase $\mathrm{K}$ (PK) for 2 days with a mixture of lung tissue homogenates and CNMs showed an average recovery rate over $90 \%$. The utility of this method was confirmed in a murine pharyngeal aspiration model using CNMs at $30 \mu \mathrm{g} /$ mouse.

Conclusions: We developed an improved lung burden assay for CNMs with an accuracy > 94\% and a recovery rate $>90 \%$ using PK digestion and UV-Vis spectrophotometry. This method can be applied to any nanomaterial with sufficient absorbance in the near-infrared band and can differentiate nanomaterials from elements in the body, as well as the soluble fraction of the nanomaterial. Furthermore, a combination of PK digestion and other instrumental analysis specific to the nanomaterial can be applied to organ burden analysis.

Keywords: Carbon black, Nanodiamond, Multi-walled carbon nanotube, Carbon nanofiber, Graphene, Lung burden

\section{Background}

Inhalation is the most common and hazardous route of exposure to nanomaterials in an occupational setting. Inhalation of nanomaterials produces a higher deposition rate of the micron-sized particles within the alveoli as a result of their size-dependent aerodynamic properties [1-3]. Furthermore, deposited particles exhibit limited clearance rates from the alveoli due to the absence of

\footnotetext{
* Correspondence: wcho@dau.ac.kr

'Lab of Toxicology, Department of Health Sciences, Dong-A University, 37, Nakdong-daero 550 beon-gil, Saha-gu, Busan 49315, Republic of Korea Full list of author information is available at the end of the article
}

mucociliary clearance. The clearance of these nanomaterials from the alveoli is influenced by the physicochemical properties of the material including size, shape, functionalization, and dissolution [4-6]. Because of the long retention period for nanomaterials in the lungs, the Organization for Economic Cooperation and Development (OECD) testing guidelines call for repeated inhalation studies (i.e., TG 412 and 413 ) and were revised in 2018 to include lung burden measurements showing lung clearance kinetics for the material of interest $[7,8]$.

There are various methods which can be used to measure the lung burden of non-labelled nanomaterials. Generally, lung burden analysis can be divided into two 
steps: (1) collection of nanomaterials from the lung and (2) quantification of nanomaterials using instrumental analysis. In the first step, chemical or enzymatic digestion methods are commonly used to collect nanomaterials from the lung tissue. Chemical digestion methods using acids, alkalis, and oxidants are all common but chemical digestion reagents can damage the structure of the nanomaterials resulting in defects, dissolution and oxidation [9]. Enzymatic digestion uses proteinase or collagenase with a chemical lysis buffer and has been proposed as an alternative to chemical lysis, as this degradation approach seems to limit structural damage of the nanomaterials $[9,10]$. In the second step, nanomaterials can be measured by various instrumental analyses including inductively coupled plasma mass spectrometry (ICP-MS), fluorometry, and optical absorbance spectrometry. For carbon nanomaterials (CNMs), the determination of the concentration is challenging because of the difficulty of measuring carbon in an organic matrix. Several approaches have been used to measure CNMs in biological matrices, including gel electrophoresis [11], programmed thermal analysis (PTA) [9], Raman spectroscopy [12], and near-infrared (NIR) spectroscopy [13]. However, there are calls for the development of more efficient and reliable measurement methods or protocols for CNMs in an organ.
Carbon nanomaterials (CNMs) such as carbon nanotubes, graphene and carbon black are considered hazardous materials when inhaled because of their biopersistence, high bio-durability, and unique physicochemical properties including their size and shape [1417]. Therefore, the precise evaluation of the kinetics of CNMs is required for proper hazard and risk assessment of CNMs. In this study, we developed an efficient and reliable protocol for measuring the lung burden of various CNMs including carbon black $(\mathrm{CB})$, nanodiamond (ND), multi-walled carbon nanotube (MWCN $\mathrm{T})$, carbon nanofiber (CNF), and graphene nanoplatelet (GNP) using proteinase $\mathrm{K}$ (PK) tissue digestion and quantification of the recovered CNMs using a UV-Vis spectrophotometer.

\section{Results}

\section{Working scheme}

Figure 1 is a schematic of the workflow used to evaluate CNM lung burden in this study. Five types of CNM including $\mathrm{CB}, \mathrm{ND}, \mathrm{MWCNT}, \mathrm{CNF}$, and GNP were selected as test materials, which allowed us to cover most of the available CNMs currently employed in research and industries. The first step in our assay development was to identify the optimal wavelength for measuring CNM concentration. This wavelength is needed to reduce any interference from the biocomponents of the

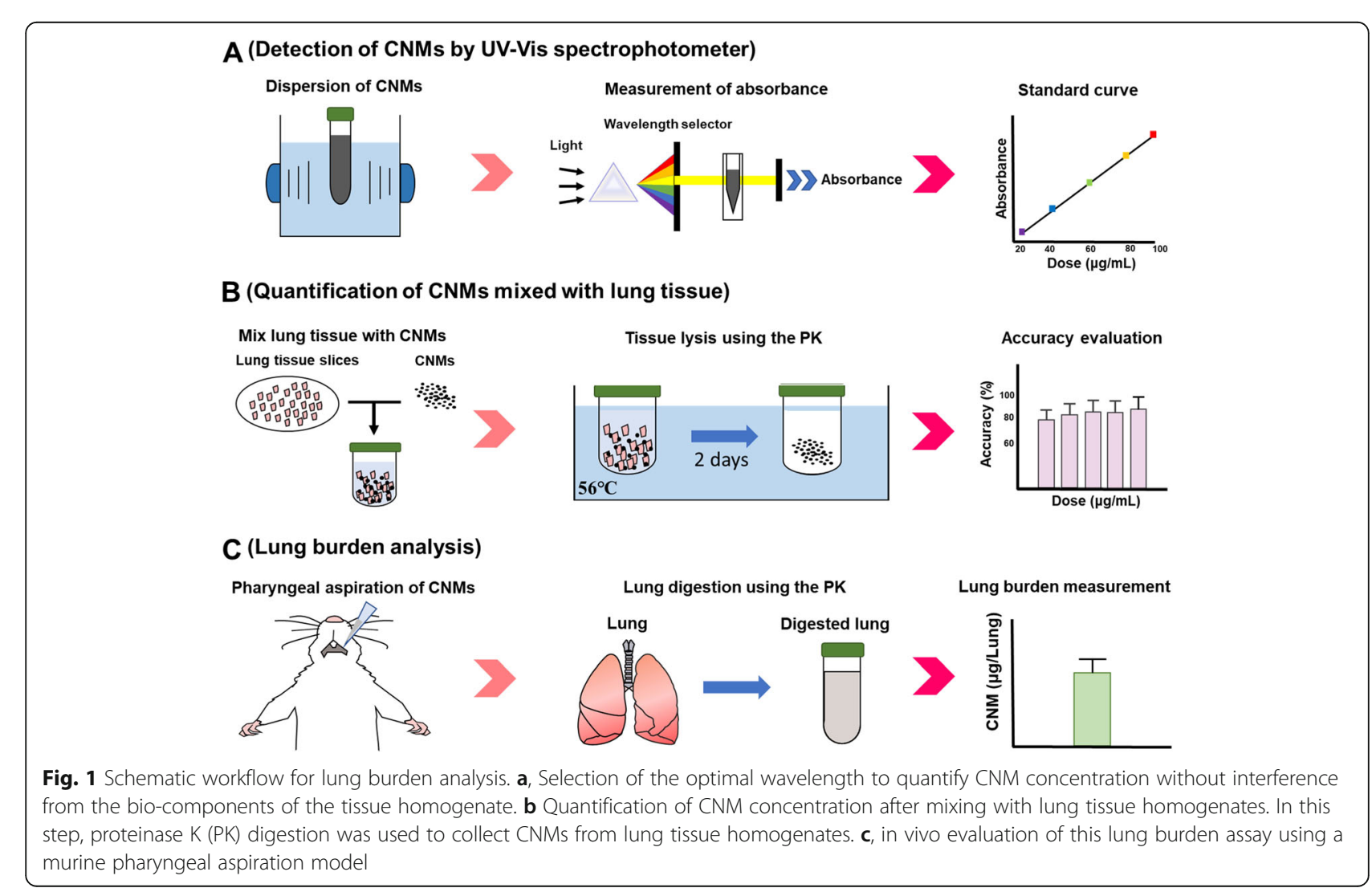


lung homogenates while still giving accurate CNM quantitation (Fig. 1a). The second step was evaluating the quantification of CNMs after they were added to the lung tissue homogenates (Fig. 1b). To do this CNMs were collected from lung tissue homogenates following PK digestion. Finally, we needed to evaluate this assay in an in vivo model; here we measured CNM lung burden at $24 \mathrm{~h}$ post pharyngeal aspiration in mice (Fig. 1c).

Transmission electron microscopy (TEM) analysis of CNMs Representative TEM images of the CNMs are presented in Fig. 2. CB and ND were spherical with an average size of $14 \pm 0.2 \mathrm{~nm}$ and $4.87 \pm 0.4 \mathrm{~nm}$, respectively. MWCNT and CNF were tubular. The size, specific surface area, and IG/ID ratio of CNMs are presented in Table 1. The diameter and length of MWCNT were $16.7 \pm 0.2 \mathrm{~nm}$ and $3.55 \mu \mathrm{m}$, respectively. The diameter and length of CNF were $24.79 \pm 0.4 \mathrm{~nm}$ and $<10 \mu \mathrm{m}$, respectively. GNP was plate-shaped with a mean diameter of $512 \pm 9.7 \mathrm{~nm}$. The BET specific surface area of CNMs was ranged 184-500 $\mathrm{m}^{2} / \mathrm{g}$. The ID/IG ratio of CNMs showed variable values by the types of CNMs.

\section{Optimal wavelength selection for measuring CNM concentration with minimal interference}

CNMs dispersed in distilled water (DW) with $3 \%$ foetal bovine serum (FBS) were shown to have increased absorbance in the $200-300 \mathrm{~nm}$ range, which then reached a plateau and stabilised until absorbance reached 900 nm (Fig. 3a). The absorbance of the empty vehicle (DW with $3 \% \mathrm{FBS}$ ) used in this study also showed increasing absorbance between 200 and $300 \mathrm{~nm}$, but its absorbance was reduced to nearly zero after $500 \mathrm{~nm}$. In addition, CNMs/lung homogenate mixtures exhibited slightly higher absorbance values from 200 to $900 \mathrm{~nm}$ when compared to CNMs in DW with 3\% FBS (Fig. 3b). However, the absorbance of the lung tissue lysis solution was shown to be reduced after $750 \mathrm{~nm}$ (approximately 0.068), which meant that this wavelength could be used to successfully measure CNMs in mixed biological solutions without interference. $\mathrm{CNM}$ at $25 \mu \mathrm{g} / \mathrm{mL}$ were shown to have an absorbance of 0.219 at $750 \mathrm{~nm}$ (Fig. $3 \mathrm{~b}$ ), confirming that $750 \mathrm{~nm}$ was the optimal wavelength for evaluations of CNM concentration in lung tissue homogenates. Because this technique uses optical absorbance in the near-infrared region, any nanomaterials having a strong absorbance in this range could be quantified using a similar approach.

\section{Quantification of CNMs dispersed in DW with 3\% FBS}

To evaluate the detection limit for CNMs, a range of CNM concentrations ( 0 to $1000 \mu \mathrm{g} / \mathrm{mL}$ ) were resuspended in DW with 3\% FBS and then subjected to evaluation at $750 \mathrm{~nm}$. The lower and upper detection limits for a linear dose-response were $0.39-50 \mu \mathrm{g} / \mathrm{mL}$ for $\mathrm{CB}$, MWCNT, and CNF, and 1.56-200 $\mu \mathrm{g} / \mathrm{mL}$ for ND and GNP (Fig. S1, see Supporting Information). To evaluate the accuracy and reproducibility of this detection method, four concentrations of each of the CNMs (i.e., 10, 20, 30, and $300 \mu \mathrm{g} /$ $\mathrm{mL}$ ) were tested. The $\mathrm{R}^{2}$ values of standard curve fits for all CNMs were more than 0.98 (Fig. 4). The detection accuracy (\%) for all CNMs was more than $94 \%$ compared to the target concentration (Table 2).

\section{Quantification of CNMs from the lung tissue homogenates}

The second step in developing our lung burden assay was to evaluate its efficacy in a tissue setting. To do this, $\mathrm{CNMs}$ were mixed with lung tissue homogenates, treated with PK and then evaluated using the UV-Vis spectrophotometer technique described above. First
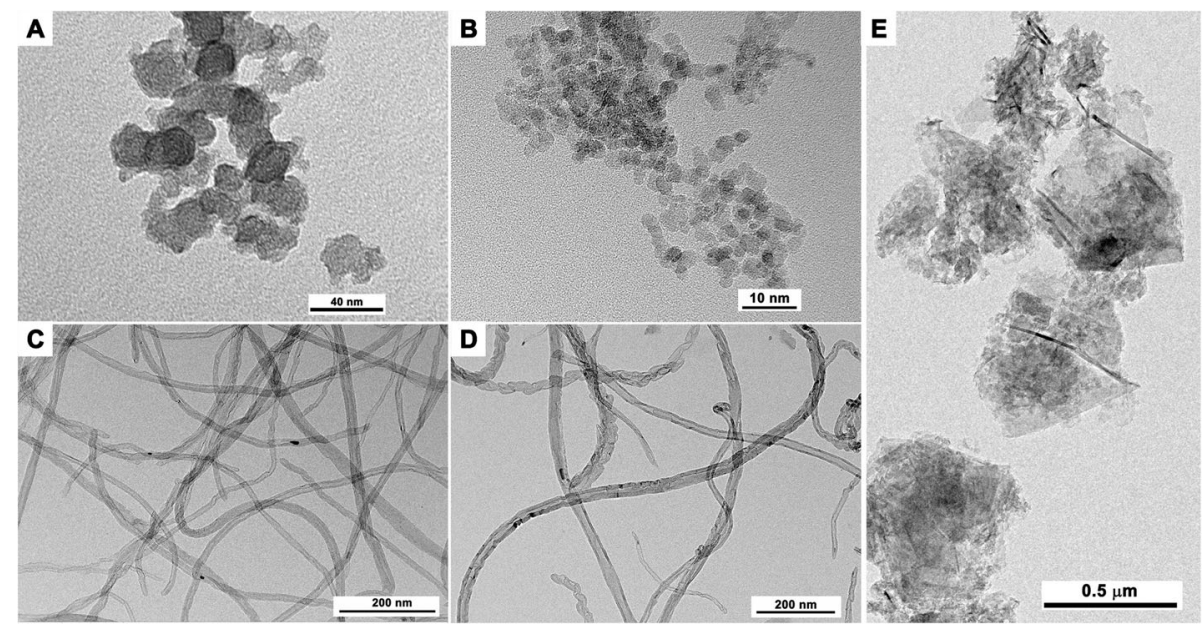

Fig. 2 The shape and morphology of various CNMs evaluated using transmission electron microscopy. $\mathbf{a}$, carbon black; $\mathbf{b}$, nanodiamonds; $\mathbf{c}$, carbon nanotube; $\mathbf{d}$, carbon nanofiber; $\mathbf{e}$, graphene nanoplatelet 
Table 1 The physicochemical properties of CNMs

\begin{tabular}{llll}
\hline CNMs & Size & Surface area $\left(\mathbf{m}^{\mathbf{2} / \mathbf{g})}\right.$ & Raman $($ ID/IG) \\
\hline CB & $14 \pm 0.2 \mathrm{~nm}$ & 254 & 0.35 \\
ND & $4.87 \pm 0.4 \mathrm{~nm}$ & 279 & 0.3 \\
MWCNT & Diameter: $16.7 \pm 0.2 \mathrm{~nm}$ & 224 & 1.46 \\
& Length: $3.55 \mu \mathrm{m}$ & & 1.02 \\
CNF & Diameter: $24.79 \pm 0.4 \mathrm{~nm}$ & 184 & 1.43 \\
& Length: $<10 \mu \mathrm{m}$ & 500 & \\
GNP & $512 \pm 9.7 \mathrm{~nm}$ & 5 & \\
\hline
\end{tabular}

$0.02 \mathrm{~g}$ (dry weight) of lung tissue homogenates were treated with $1 \mathrm{~mL}$ of Tris buffer ( $\mathrm{pH} 8.0$ ) containing $200 \mu \mathrm{g}$ PK at $56^{\circ} \mathrm{C}$ and showed complete lysis within 2 days (Fig. 5). The presence of erythrocytes did not influence the efficacy of the PK digestion as lung tissues were completely digested regardless of perfusion (Fig. 5). Thus, the main experiment was performed with lung tissues without perfusion. All CNMs were properly detected using this technique and the recovery percentage for the CNMs between 3.1 to $100 \mu \mathrm{g} / \mathrm{mL}$ was over $86 \%$ and mean recovery percentage of tested concentrations was over 90\% for all types of CNMs (Fig. 6 and Tables S1 and S2, see Supporting Information). It is worth noting that the UV-Vis spectrophotometer can detect both higher and lower CNM concentrations than the ones used here supporting its widespread utility in this type of application. The loss rate of CNMs by mechanical processes such as washing and centrifugation was ranged about $3-7 \%$ (Table 3). Because the loss rate was dependent on the concentration of samples, it was slightly increased as increasing the concentration.

\section{Lung burden analysis after pharyngeal aspiration of CNMs in mice}

As a pilot study, we evaluated the deposition rate at time zero and retention rate at $24 \mathrm{~h}$ after a single pharyngeal aspiration of CNMs. The deposition rates of $\mathrm{CB}, \mathrm{ND}$, MWCNT, CNF, and GNP at time zero compared to the nominal treatment dose $(30 \mu \mathrm{g} /$ mouse $)$ were $84.9,80.4$, $79.1,80.2$, and $75.7 \%$, respectively (Fig. 7a). While, the retention rates of $\mathrm{CB}, \mathrm{ND}, \mathrm{MWCNT}, \mathrm{CNF}$, and GNP at $24 \mathrm{~h}$ after aspiration compared to that of time zero were 99.5, 99.5, 98.4, 97.9, and 97.5\%, respectively (Fig. 7b).

\section{Discussion}

Measuring the lung burden of nanomaterials is now mandatory under the revised inhalation toxicity testing guidelines (i.e., TG412 and TG413) published by the OECD $[7,8]$, and there is an ongoing project of testing guidelines for toxicokinetics to accommodate nanomaterials [18]. In addition, new or improved methodologies to evaluate the concentration of nanomaterials in biological samples is essential for continued research including evaluating nanomaterials in biomedical applications. Thus, this study was designed to create a novel methodology to quantify CNMs deposited in the lung using PK enzymatic digestion and UV-Vis spectrophotometry.

The recovery of CNMs from organ tissue homogenates is critical for the success of organ burden assays and various chemical cocktails or enzymes have been suggested for the digestion of these organ tissues [9].
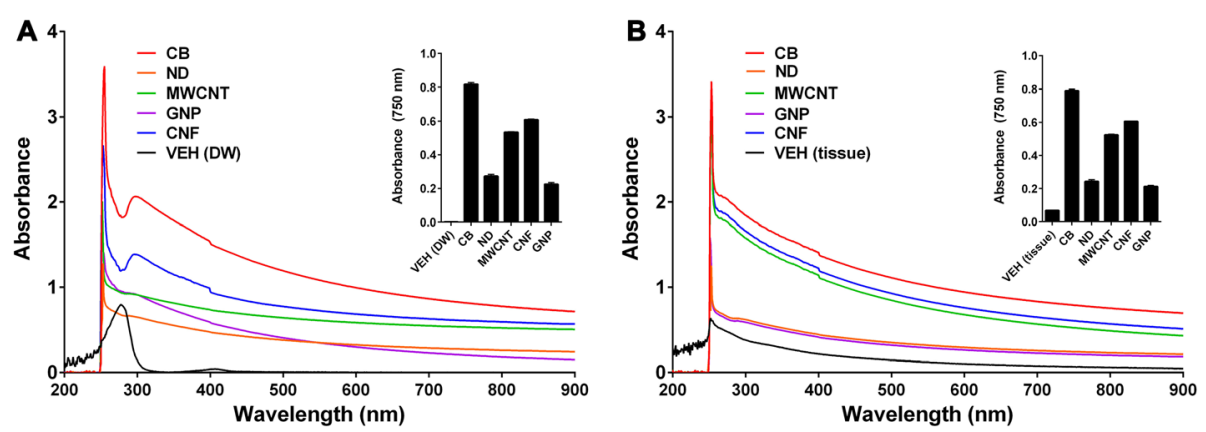

Fig. 3 The selection of an optimal wavelength to quantify CNMs without interference from tissue homogenates. a, Absorbance of CNMs or vehicle control [VEH (DW), DW with 3\% FBS]. b, Absorbance of vehicle control [VEH (tissue), tissue lysates in DW] and CNMs collected from a mixture of $25 \mu \mathrm{g} / \mathrm{mL}$ CNMs and $0.02 \mathrm{~g}$ (dry weight) lung tissue homogenates following treatment with $200 \mu \mathrm{g}$ of proteinase $\mathrm{K}$ (PK). The insert figure represents that the absorbance of the bio-components was lower at $750 \mathrm{~nm}$ after treatment with PK, making it the optimal wavelength to quantify CNMs without interference from the tissue homogenate 

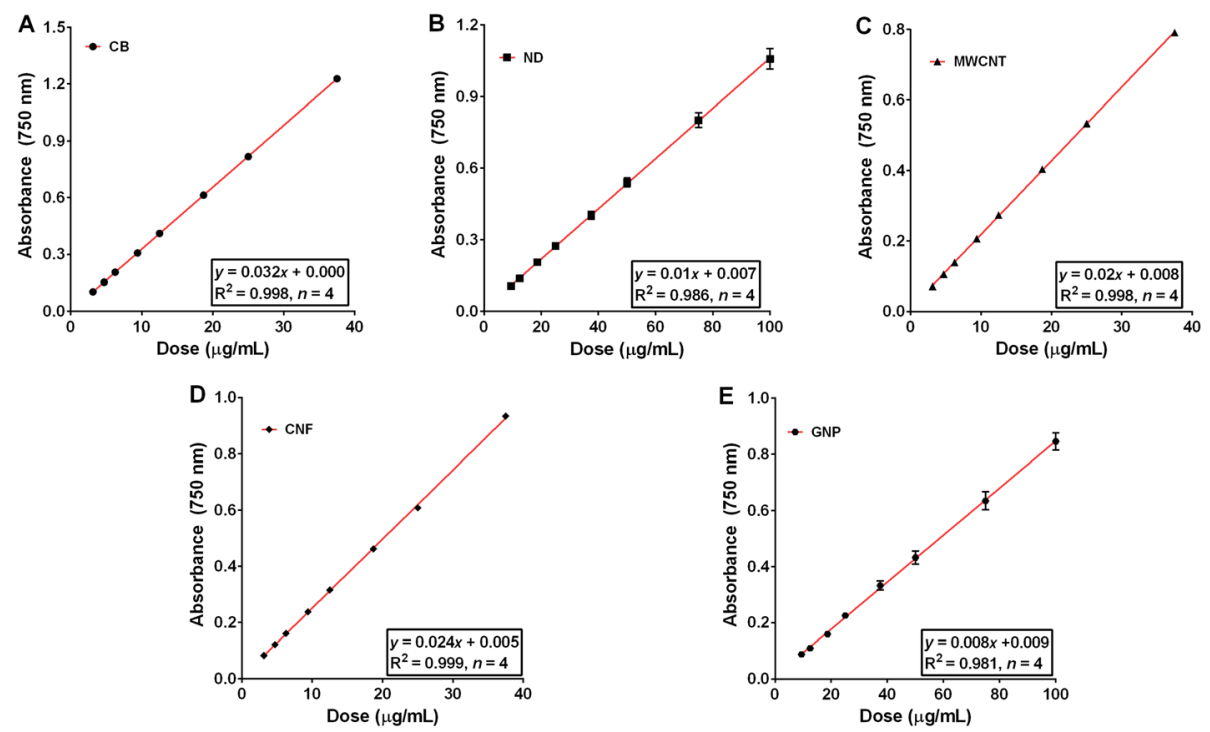

Fig. 4 The standard curve fit of CNMs using a UV-Vis spectrophotometer. CNMs were dispersed in distilled water with 3\% foetal bovine serum and absorbance was measured at $750 \mathrm{~nm}$. a, carbon black (CB); b, nanodiamond (ND); c, multi-walled carbon nanotube (MWCNT); d, carbon nanofiber (CNF); e, graphene nanoplatelet (GNP). Data are expressed as mean \pm SEM and $n=4$

Chemical cocktails to digest organ tissues use oxidants, acids, and alkalis. These cocktails are hazardous to human health and some chemicals like nitric acid can induce defects or degradation of nanomaterials even in their most stable formats [19]. Because CNMs are

Table 2 The accuracy (\%) of the UV-Vis spectrophotometer technique when measuring CNM concentration

\begin{tabular}{lllll}
\hline CNMs & \multicolumn{4}{l}{ Target concentration $(\boldsymbol{\mu g} / \mathbf{m L})$} \\
\cline { 2 - 5 } & $\mathbf{1 0}$ & $\mathbf{2 0}$ & $\mathbf{3 0}$ & $\mathbf{3 0 0}^{\mathbf{a}}$ \\
\hline CB & & & \\
Measured & $9.53 \pm 0.28$ & $19.37 \pm 0.66$ & $28.76 \pm 0.47$ & $285.49 \pm 1.04$ \\
Accuracy \% & $95.3 \pm 2.84$ & $96.8 \pm 3.33$ & $95.8 \pm 1.57$ & $95.1 \pm 0.34$ \\
ND & & & \\
Measured & $9.51 \pm 0.26$ & $19.07 \pm 0.76$ & $28.52 \pm 1.03$ & $286.06 \pm 4.05$ \\
Accuracy \% & $95.1 \pm 2.61$ & $95.3 \pm 3.82$ & $95.0 \pm 3.43$ & $95.3 \pm 1.35$ \\
MWCNT & & & & \\
Measured & $9.69 \pm 0.18$ & $19.29 \pm 0.57$ & $28.75 \pm 1.11$ & $285.12 \pm 4.55$ \\
Accuracy \% & $96.9 \pm 1.84$ & $96.4 \pm 2.85$ & $95.8 \pm 3.70$ & $95.0 \pm 1.51$ \\
CNF & & & & \\
Measured & $9.42 \pm 0.56$ & $19.03 \pm 0.57$ & $28.69 \pm 1.06$ & $286.04 \pm 2.22$ \\
Accuracy \% & $94.2 \pm 5.67$ & $95.1 \pm 2.88$ & $95.6 \pm 3.55$ & $95.3 \pm 0.74$ \\
GNP & & & & \\
Measured & $9.63 \pm 0.11$ & $19.48 \pm 0.87$ & $29.22 \pm 1.10$ & $289.88 \pm 9.92$ \\
Accuracy \% & $96.2 \pm 1.12$ & $97.4 \pm 4.37$ & $97.4 \pm 3.68$ & $96.6 \pm 3.30$ \\
\hline
\end{tabular}

The accuracy (\%) was calculated by comparison with the target concentration weighed during sample preparation

${ }^{\text {aT }}$ The target concentrations were selected as 10,20 , and $30 \mu \mathrm{g} / \mathrm{mL}$ for nondiluted samples and $300 \mu \mathrm{g} / \mathrm{mL}$ for samples needed dilution

The data are presented as mean \pm SEM from four independent measurements commonly quantified using thermal or optical methods structural defects or degradation of CNMs can result in inaccurate quantification. In addition, most organ burden analysis of metal-based nanomaterials use acid digestions to lyse the organ tissues and nanomaterials [20, 21]. However, this method cannot discriminate between nanomaterial-derived metal ions and tissue-derived metals or bio-persistent nanomaterials from dissolved ions $[22,23]$. For example, the iron concentration collected by acid digestion of organs treated with iron oxide nanomaterials can be derived from iron in the organ, iron from dissolved iron oxide in the body fluid such as lysosomal fluid, or iron from bio-persistent iron oxide. Thus, the extraction of nanomaterials from the organ without defect or degradation is critical for the accurate quantification of nanomaterials in organ tissues. The enzymatic digestion of these tissues could therefore provide a solution to these problems. Here, we showed that enzymatic digestion could facilitate the recovery of nanomaterials from tissue homogenate without damaging the CNMs and allowed for the evaluation of morphological changes like defects or biotransformation [24].

Mineralization of CNMs is required for the acid digestion methods such as $\mathrm{HCl}$, nitric acid, and hydrofluoric acid. However, some of the chemical digestion agents such as Solvable (PerkinElmer, Waltham, MA, USA) and Clean 99-K200 (Clean Chemical, Osaka, Japan) and enzymatic digestion methods do not require the mineralization process $[9,25]$. Because the mineralization process can reduce the recovery of CNMs, the wet process such as proteinase-K can have advantages to collect CNMs in the biological matrices. To our knowledge, this the first report 


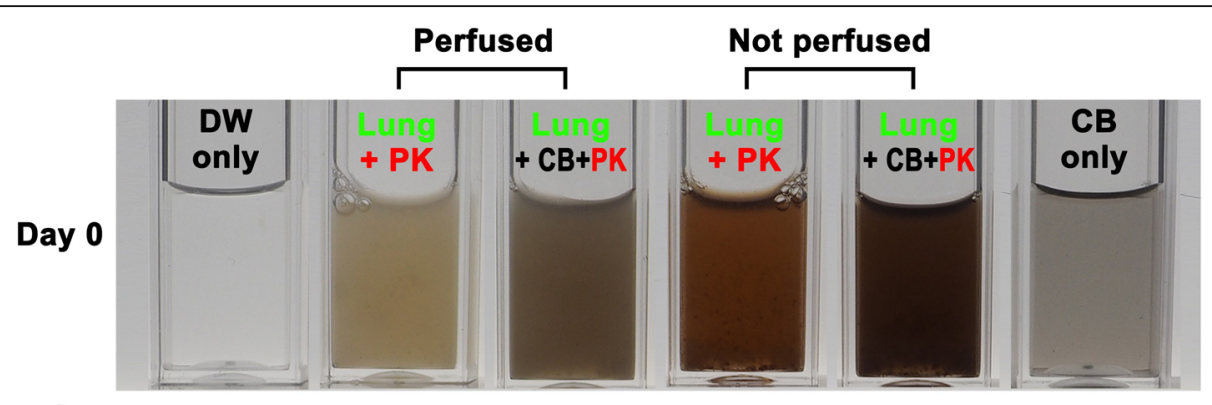

$\downarrow$ Washing by centrifugation and resuspension
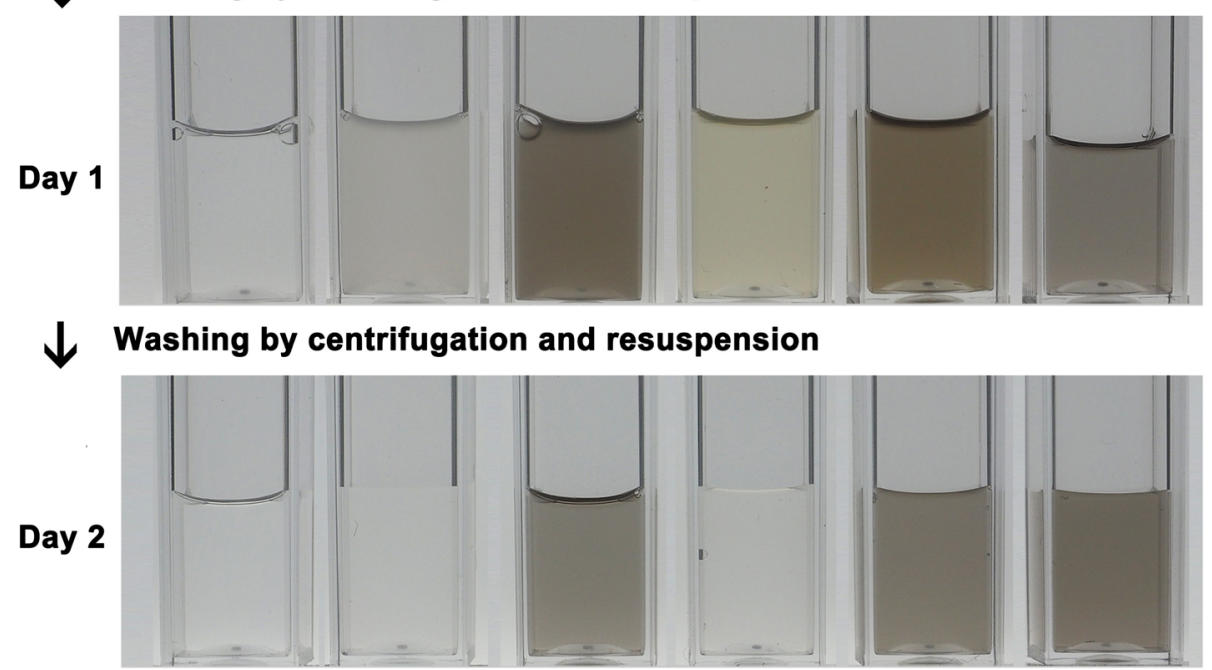

Fig. 5 The visual changes in the lung tissue homogenates after incubation with proteinase K (PK). Carbon black (CB) was selected as a representative CNM and a mixture of $\mathrm{CB}$ at $25 \mu \mathrm{g}$ and lung tissue homogenates at $0.02 \mathrm{~g}$ (dry weight) were incubated in $1 \mathrm{~mL}$ of Tris buffer ( $\mathrm{pH}$ 8.0) containing $200 \mu \mathrm{g}$ PK. After $24 \mathrm{~h}$ samples were washed by centrifugation and incubated for another $24 \mathrm{~h}$ in $1 \mathrm{~mL}$ of Tris buffer ( $\mathrm{pH}$ 8.0) containing $200 \mu \mathrm{g}$ PK. Note that CB could be completely recovered from the lung tissue homogenates following PK digestion and centrifugation. The perfusion step did not influence nanomaterial recovery

demonstrating the recovery of CNMs from the mixture of tissue homogenates and nanomaterials using the PK digestion method. Here, we were able to recover about $90 \%$ of the CNMs mixed with lung tissue homogenates. The loss of CNMs during the lung burden analysis using PK digestion method can be due to the mechanical loss (e.g., 3-7\%) such as washing and centrifugation and inaccuracy (e.g., $<6 \%$ ) of the UV-Vis spectrophotometer technique as shown in this study. Furthermore, PK enzymatic digestion was shown to work perfectly with or without perfusion, which is advantageous for toxicity studies.

After collecting nanomaterials from the organ tissue, it is important to select an appropriate method for instrumental analysis; this will allow proper quantification of the relevant material. CNMs are generally measured using programmed thermal analysis (PTA) like NIOSH $5040[5,26]$. However, PTA analysis requires expensive instrumentation and inaccuracies can occur when CNMs are damaged during the extraction process. This method shows only about $50 \%$ recovery in lung burden analyses from a 28-day inhalation study of MWCNT [5, 9]. Here, we have been able to show that UV-Vis spectrophotometry can accurately measure five types of CNM over a range of concentrations $(0.39-50 \mu \mathrm{g} / \mathrm{mL}$ for $\mathrm{CB}, \mathrm{MWCNT}$, and CNF; $1.56-200 \mu \mathrm{g} / \mathrm{mL}$ for $\mathrm{ND}$ and GNP) with more than 94\% accuracy. Although this study showed a detection limit of MWCNT as $0.39 \mu \mathrm{g} / \mathrm{mL}$, a previous study suggests that the limit of detection for MWCNT using a UV-Vis spectrophotometer is $0.025 \mu \mathrm{g} / \mathrm{mL}$ [10], which is more sensitive than any of the metallabelling methods like Ni-labelling, which showed a $0.1 \mu \mathrm{g} / \mathrm{mL}$ limit of detection [27], and PTA, which showed $0.2 \mu \mathrm{g}$ limit of detection [9]. In comparison with this study, the lower detection limit of MWCNT performed by Zhang et al. [10] could be due to the difference in the physicochemical properties such as length, diameter, and dispersibility [28-30]. Furthermore, the use of UV-Vis spectrophotometer is available to other types of nanomaterials such as metal oxides (Table S3, see Supporting Information). 

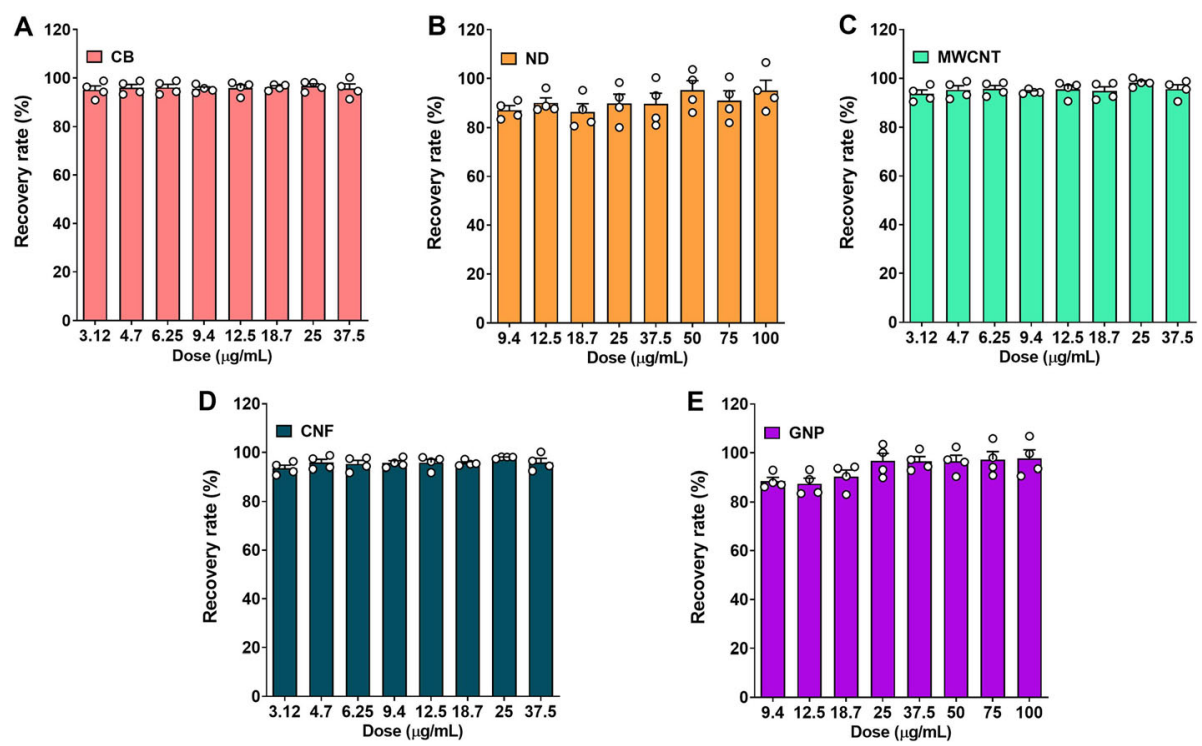

Fig. 6 The recovery rate of CNMs mixed with lung tissue homogenates following proteinase K (PK) digestion. All CNMs in the tested dose ranges showed a more than $86 \%$ recovery rate from lung tissue homogenates following PK digestion. $\mathbf{a}$, carbon black; $\mathbf{b}$, nanodiamond; $\mathbf{c}$, carbon nanotube; $\mathbf{d}$, carbon nanofiber; e, graphene nanoplatelet. Data are expressed as mean \pm SEM and $n=4$. The detailed numeric data are presented in Tables S1 and S2 (see Supporting Information)

\section{Conclusions}

In this study, we developed an optimized lung burden assay with over $90 \%$ accuracy and $83 \%$ recovery rates designed to evaluate CNMs. This methodology relies on a PK digestion and UV-Vis spectrophotometry. This method can also be applied to other nanomaterials with significant absorbance in the near-infrared band. In addition, this technique can be applied to differentiate between the nanomaterials from the elements of the body or soluble fraction. Furthermore, the combination of PK digestion and other instrumental analysis, such as PTA, ICP-MS, fluorometer, or particle counter, could help to overcome the limitations in quantifying other nanomaterials in biological samples.

\section{Materials and methods}

\section{Selection of CNMs and TEM analysis}

A panel of CNMs was assembled to include various types of nanomaterials including $\mathrm{CB}$, ND, MWCNT,

Table 3 The loss rate (\%) of CNMs by mechanical processes such as washing and centrifugation

\begin{tabular}{llll}
\hline Nominal Dose $(\boldsymbol{\mu} \mathbf{g} / \mathbf{m L})$ & $\mathbf{1 0}$ & $\mathbf{2 0}$ & $\mathbf{3 0}$ \\
\hline CB & $4.60 \pm 0.78^{\text {a }}$ & $4.84 \pm 1.14$ & $4.60 \pm 0.68$ \\
ND & $4.19 \pm 1.06$ & $3.95 \pm 1.27$ & $3.87 \pm 0.44$ \\
MWCNT & $6.20 \pm 0.71$ & $5.28 \pm 0.94$ & $4.54 \pm 0.79$ \\
CNF & $5.73 \pm 1.70$ & $4.25 \pm 0.99$ & $4.11 \pm 0.75$ \\
GNP & $4.97 \pm 1.15$ & $4.81 \pm 1.14$ & $4.26 \pm 1.91$ \\
\hline
\end{tabular}

${ }^{a}$ The recovered concentration was divided by the initial dose to calculate the loss rate

Data are mean \pm SEM and $n=4$ for each concentration
CNF, and GNP. Based on the morphology, two materials can be classified as particles (i.e., CB and ND), two as fibers (i.e., MWCNT and CNF), and one as a platelet (i.e., GNP). All CNMs were provided from commercial sources: CB (\# Printex 90; Evonik Degussa GmbH, Frankfurt, Germany), ND (\# ND1; S.W. Chemicals Co., Ltd., Gunsan, Korea), MWCNT (\#CM-100; Hanwha Nanotech Co., Seoul, Korea), CNF (\# T-CNF; Carbon Nano-material Technology Co., Pohang, Korea), and graphene (\# 06-0230; Stream Chemical Inc., Newburyport, MA, USA). The size and shape of the CNMs were evaluated by TEM (JEM-1200EXII, JEOL, Tokyo, Japan) as described in our previous study [31]. The size of the CNMs was calculated by counting at least $300 \mathrm{CNMs}$ using a built-in analysis program (JEOL). Raman spectroscopy was used on the CNMs to evaluate defects using a WITec alpha300 system (WITec GmbH, Ulm, Germany) with incident laser light at a wavelength of $532 \mathrm{~nm}$. The surface area of the CNMs was measured with the Brunauer-Emmett-Teller method using a BELSORP-mini II (BEL Japan Inc.).

\section{Dispersion of CNMs}

The degree of dispersion of the preparations is critical because NIR signals are known to be dispersiondependent $[29,30]$. Because of the hydrophobic nature of the CNMs, serum was used as the dispersion medium to provide a protein corona, which ensured proper dispersion of the CNMs within the media [32]. Furthermore, a water bath sonicator was applied to breakup 

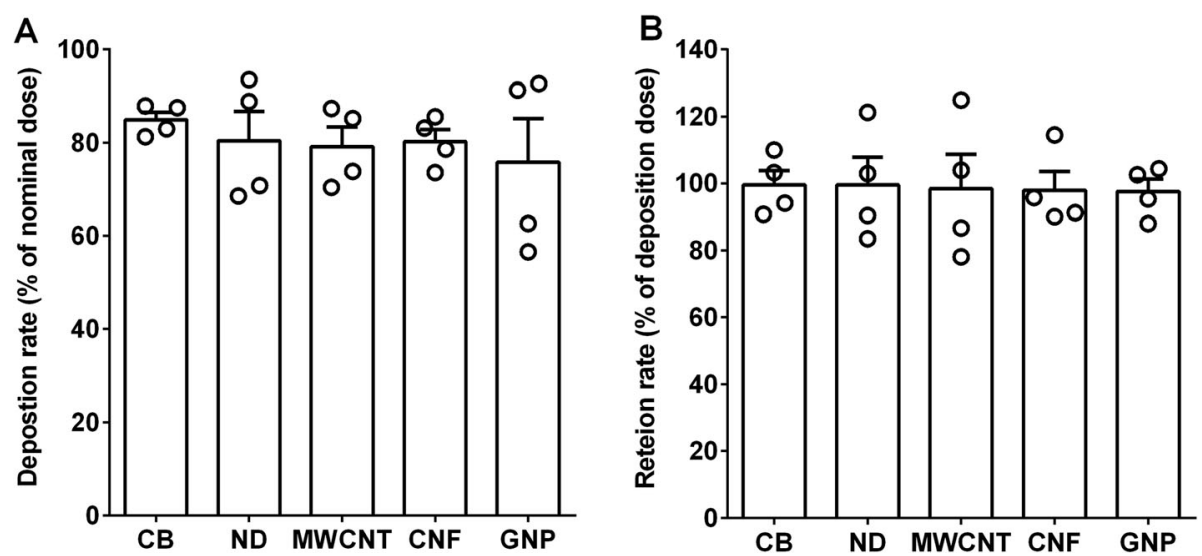

Fig. 7 A pilot study to evaluate the lung deposition rate (a) and retention rate (b) of CNMs. To evaluate the deposition rate in comparison with the nominal treatment dose, mice were sacrificed immediately after a single pharyngeal aspiration and lung burden analysis was performed. To evaluate the retention rate of CNMs at $24 \mathrm{~h}$, mice were sacrificed at $24 \mathrm{~h}$ after a pharyngeal aspiration. Mice were given $30 \mu \mathrm{g} / \mathrm{mouse}$ of CNMs and the concentration of these CNMs in the lungs was quantified using PK digestion and UV-Vis spectrophotometry at each time-point. Data are expressed as mean \pm SEM and $n=4$

agglomerates, which was broadly used in the process of nanomaterial dispersion [30, 33, 34]. Briefly, CNM powders were dispersed in DW containing 30\% $v / v$ heatinactivated FBS and sonicated using a bath sonicator (Saehan Sonic, Seoul, Korea) to break up agglomerates. The operation condition of a bath sonicator was $40 \mathrm{kHz}$ frequency and $400 \mathrm{~W}$ output power. Then, DW was added to make up the final working solution of CNMs, and the concentration of FBS was kept to less than 3\% $v / v$. The target concentrations of the stock solution and working solution to evaluate the dispersion of CNM was $1 \mathrm{mg} / \mathrm{mL}$ and $25 \mu \mathrm{g} / \mathrm{mL}$, respectively. The dispersibility of stock solution was measured by optical absorbance at $750 \mathrm{~nm}$ after diluting with DW at $25 \mu \mathrm{g} / \mathrm{mL}$. After the selected optimal sonication duration of stock solution, the working solution was prepared at $25 \mu \mathrm{g} / \mathrm{mL}$ in DW and the dispersibility was evaluated after further sonication for 10-30 min. Because each CNMs needs a nanomaterial-specific duration of sonication for the best dispersion efficacy, the stock solution and working solution were sonicated with different durations (Table 4 and Fig. S2). All CNMs were stable up to $4 \mathrm{~h}$ with some minor variations between types of CNMs (Table 4 and Fig. S3). All standards and samples were measured within $10 \mathrm{~min}$ after the dispersion process.

Table 4 Duration of sonication for the best dispersion of CNMs

\begin{tabular}{lcclll}
\hline CNMs & CB & ND & MWCNT & CNF & GNP \\
\hline Duration of sonication & & & & \\
Stock & $80 \mathrm{~min}$ & $10 \mathrm{~min}$ & $80 \mathrm{~min}$ & $80 \mathrm{~min}$ & $30 \mathrm{~min}$ \\
Working & $10 \mathrm{~min}$ & $10 \mathrm{~min}$ & $10 \mathrm{~min}$ & $10 \mathrm{~min}$ & $10 \mathrm{~min}$ \\
$\begin{array}{l}\text { Duration of the dispersion stability } \\
\text { Working }\end{array}$ & $4 \mathrm{~h}$ & $16 \mathrm{~h}$ & $4 \mathrm{~h}$ & $4 \mathrm{~h}$ & $4 \mathrm{~h}$ \\
\hline
\end{tabular}

\section{Measurement of CNMs dispersed in DW using a UV-Vis spectrophotometer}

To evaluate the accuracy CNM concentration measurements on the UV-Vis spectrophotometer, the absorbance spectra of well-dispersed CNMs in 3\% FBS DW were measured at $200-900 \mathrm{~nm}$ in quartz cuvettes using a UV-Vis spectrophotometer (Lambda 365, PerkinElmer, Waltham, Massachusetts, USA). Based on these results and those from several previous studies [35-37], we selected an absorption wavelength of $750 \mathrm{~nm}$ for all of the CNMs experiments. To estimate the linear dosage range for the standard curve various concentrations of CNMs from 0 to $1000 \mu \mathrm{g} / \mathrm{mL}$ were tested evaluated using the spectrophotometer, a standard curve of 8 concentrations (3.1, 4.7, 6.2, 9.4, 12.5, 18.7, 25, and $37.5 \mu \mathrm{g} /$ $\mathrm{mL}$ for $\mathrm{CB}, \mathrm{MWCNT}$, and CNF; 9.4, 12.5, 18.7, 25, 37.5, 50,75 , and $100 \mu \mathrm{g} / \mathrm{mL}$ for ND and GNP) was selected for further experiments. To evaluate the accuracy and reproducibility, we selected target concentrations as 10 , 20 , and $30 \mu \mathrm{g} / \mathrm{mL}$ for non-diluted samples and $300 \mu \mathrm{g} /$ $\mathrm{mL}$ for samples needed dilution. These target concentrations were not included in the data points used to calculate the calibration regression. Four independent measurements were performed for each to evaluate the accuracy and reproducibility of this system of measurement.

\section{Recovery of CNMs from lung tissue homogenates}

Six-week-old specific-pathogen-free female ICR mice were purchased from Samtako (Gyeonggi-do, Korea). The mice were maintained and handled in accordance with the procedures approved by the Institutional Animal Care and Use Committee of Dong-A University. Animals were acclimatized for one week prior to 
experimentation then they were anaesthetized with isoflurane (Piramal Critical Care, Bethlehem, PA, USA) using a VetEquip rodent anaesthesia system (Pleasanton, CA, USA) and sacrificed by exsanguination via the interior vena cava. Then, the lung was perfused via the right ventricle with pre-warmed PBS containing 3.8\% sodium citrate solution (Sigma-Aldrich, St. Louis, MO, USA). The perfused and non-perfused lungs were cut into pieces and dried in an oven at $60^{\circ} \mathrm{C}$ for $48 \mathrm{~h}$ and crushed using a tissue homogenizer (Thomas Scientific, Swedesboro, NJ, USA). A total of $0,02 \mathrm{~g}$ (dry weight) of lung tissue homogenate was mixed with the CNM mixture. Then, $1 \mathrm{~mL}$ of PK digestion buffer [ $50 \mathrm{mM}$ Tris$\mathrm{HCl}, 10 \mathrm{mM} \mathrm{CaCl}_{2}$, and $200 \mu \mathrm{g}$ PK (Promega, Madison, WI, USA), $\mathrm{pH} 8.0$ ] was added and incubated for $24 \mathrm{~h}$ at $56^{\circ} \mathrm{C}$. Samples were then centrifuged at $15000 \times g$ for 20 min and the supernatant was removed. The pellets were resuspended in $1 \mathrm{~mL}$ of PK digestion buffer and sonicated for $5 \mathrm{~min}$ in a bath sonicator (Saehan Sonic) and incubated at $56{ }^{\circ} \mathrm{C}$ for a further $24 \mathrm{~h}$. These suspensions were centrifuged at $15000 \times g$ for $20 \mathrm{~min}$ and the pellets were resuspended in $1 \mathrm{~mL}$ of DW and sonicated for 5 min in a bath sonicator (Saehan Sonic). The recovered CNMs were quantified using the UV-Vis spectrophotometer as described above.

\section{Evaluation of the loss rate of CNMs during the lung burden analysis}

We evaluated the mechanical loss rate of CNMs during various processes in the lung burden analysis such as washing and centrifugation. Before starting the experiment, the concentration of dispersed CNMs at nominal concentrations of 10,20 , and $30 \mu \mathrm{g} / \mathrm{mL}$ were measured by UV-Vis spectrophotometer. Then, the suspensions of
CNMs were processed with the identical procedures described in "Recovery of CNMs from lung tissue homogenates" without the addition of lung tissue homogenates. We excluded lung tissue homogenates to exclude the possible interference by the bio-components in the UVVis spectrophotometer technique and to focus on the mechanical loss rate of CNMs such as washing and centrifugation. Then, the recovered concentrations of CNMs were expressed as loss percentage compared to the initial concentration.

\section{Lung burden analysis after a single pharyngeal aspiration} in mice

A pilot study of lung burden analysis was performed after a single pharyngeal aspiration of CNMs in mice. A schematic of the workflow for this study is presented in Fig. 8. Six-week-old female ICR mice (Samtako) were acclimatized for one week prior to experimentation. To perform the pharyngeal aspiration, mice were anaesthetized with isoflurane (Piramal Critical Care) and placed on a board in a near-vertical position. Then, a suspensions of well-dispersed CNMs in PBS with 3\% $(v / v)$ heat-inactivated mouse serum was loaded into the mouth and aspirated by holding the tongue at full extension and covering the nose. The aspiration volume was $50 \mu \mathrm{L} /$ mouse and $3 \%$ mouse serum in PBS served as the vehicle control. The treatment dose was $30 \mu \mathrm{g} /$ mouse. To evaluate the deposition rate in comparison with the nominal treatment dose, mice were sacrificed immediately after a single pharyngeal aspiration and lung burden analysis was performed. The deposition rate was calculated by dividing the lung burden at time zero with the nominal treatment dose. To evaluate the retention rate of CNMs at $24 \mathrm{~h}$, mice were sacrificed at $24 \mathrm{~h}$ after

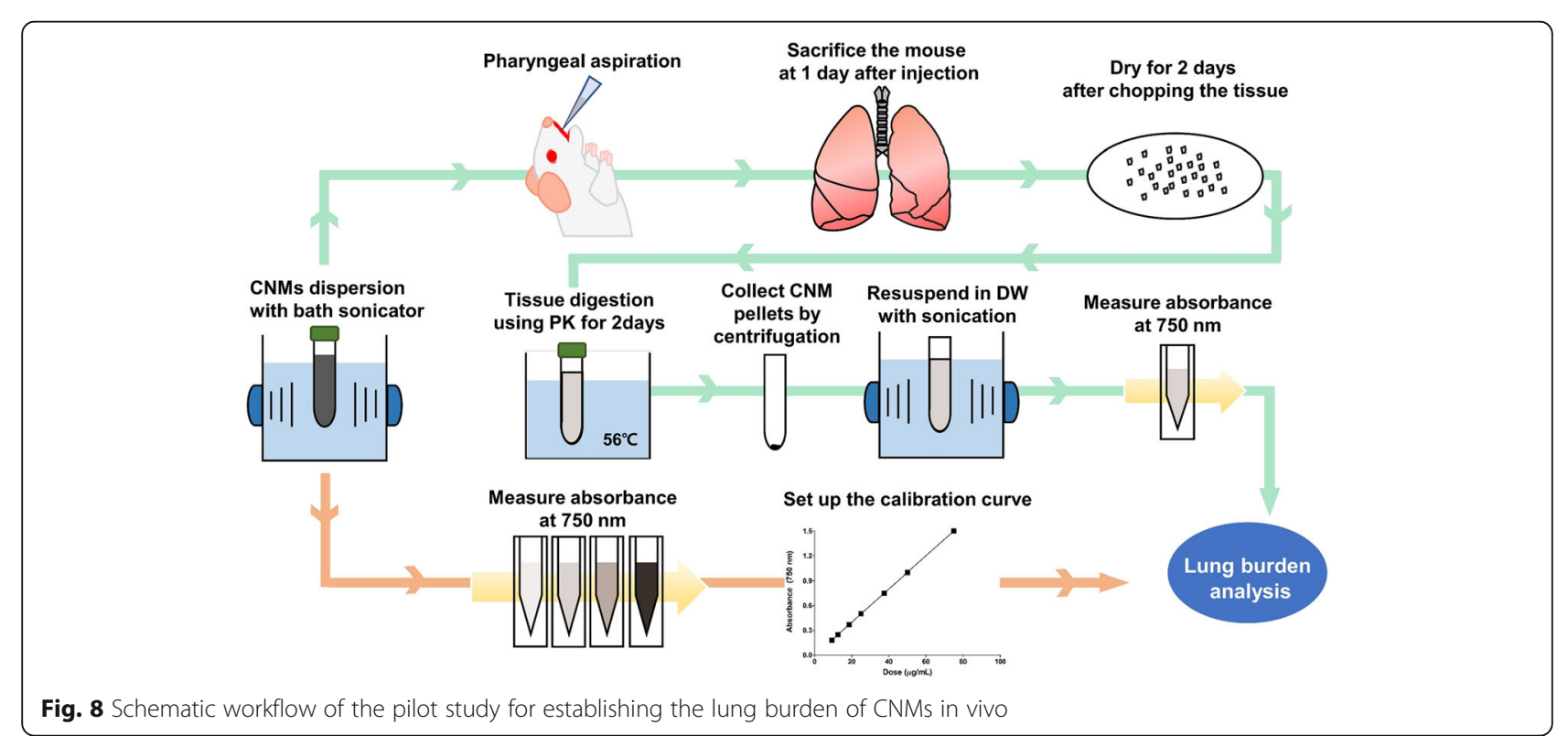


a pharyngeal aspiration. The retention rate was calculated by dividing the lung burden at $24 \mathrm{~h}$ with that of time zero. At each time-point, mice were sacrificed by removing blood from the inferior vena cava under deep isoflurane anaesthesia. Lung tissue was cut into pieces and dried in an oven at $60^{\circ} \mathrm{C}$ for $48 \mathrm{~h}$. Dried lung tissues were weighed and crushed using a tissue homogenizer (Thomas Scientific). Then, $1 \mathrm{~mL}$ of PK digestion buffer containing $200 \mu \mathrm{g}$ PK was added to $0.02 \mathrm{~g}$ (dry weight) lung homogenate and incubated for $24 \mathrm{~h}$ at $56^{\circ} \mathrm{C}$. Samples were centrifuged at $15000 \times g$ for $20 \mathrm{~min}$ to collect pellets containing CNMs and undigested lung tissue, and then resuspended in $1 \mathrm{~mL}$ of PK digestion buffer and incubated further $24 \mathrm{~h}$ at $56^{\circ} \mathrm{C}$. Finally, these samples were then sonicated for $5 \mathrm{~min}$. The CNMs were collected via centrifugation at $15000 \times g$ for 20 min and resuspended in DW with 5 min sonication. The recovered CNMs were quantified using the UV-Vis spectrophotometer as described above.

\section{Statistical analysis}

The data are presented as mean \pm SEM and linear regression was applied to the standard curve fit. GraphPad Prism software (ver. 6.0; La Jolla, CA) was used to draw the graphs and perform all the statistical analysis.

\section{Supplementary information}

Supplementary information accompanies this paper at https://doi.org/10. 1186/s12989-020-00377-9.

Additional file 1: Figure S1. Measurement of the concentration of CNMs using a UV-Vis spectrophotometer. CNMs were dispersed in distilled water with $3 \%$ FBS and tested up to $1000 \mu \mathrm{g} / \mathrm{mL}$. The absorbance was measured at $750 \mathrm{~nm}$ wavelength. Note that the lower and upper detection limits for a linear dose-response were $0.39-50 \mu \mathrm{g} / \mathrm{mL}$ for $C B$, MWCNT, and CNF, and 1.56-200 $\mu \mathrm{g} / \mathrm{mL}$ for ND and GNP. (A), carbon black; (B), nanodiamond; (C), multi-walled carbon nanotube; (D), carbon nanofiber; (E), graphene nanoplatelet. Figure S2. Evaluation of the dispersibility of CNMs. The time-course dispersibility of stock solution (A) and working solution (B) of CNMs. (A), To evaluate the dispersibility of the stock solution, $1 \mathrm{mg} / \mathrm{mL}$ stock solution was sonicated for $10 \mathrm{~min}$ 100 min. Then, at each time-point, the stock solution was diluted in DW at $25 \mu \mathrm{g} / \mathrm{mL}$ with vigorous vortexing for $30 \mathrm{~s}$ and measured the optical density at $750 \mathrm{~nm}$. (B), To evaluate the duration of sonication for working solution, the working solution $(25 \mu \mathrm{g} / \mathrm{mL})$ of each NM after an optimal sonication duration of stock solution (see Table 4) was sonicated further up to 30 min and optical density was measured at $750 \mathrm{~nm}$. $n=4$. Figure S3. Duration of the dispersion stability of CNMs. The working solution of CNMs at $25 \mu \mathrm{g} / \mathrm{mL}$ was sonicated for $10 \mathrm{~min}$ after an optimal sonication duration of stock solution (see Table 4). Then, the duration of the dispersion stability was measured at each time-point up to $24 \mathrm{~h} . n=4$. Table S1. The recovery rates of CB, MWCNT, and CNF from lung tissue homogenates following proteinase $K$ digestion with quantification using the UVVis spectrophotometer technique. Table S2. The recovery rates of ND and GNP from lung tissue homogenates following proteinase $\mathrm{K}$ digestion with quantification using the UV-Vis spectrophotometer technique. Table S3. The screening result of NIR absorbance at $750 \mathrm{~nm}$ of various types of nanomaterials.

\section{Abbreviations}

CB: Carbon black; CNF: Carbon nanofiber; CNMs: Carbon nanomaterials; MWCNT: Multi-walled carbon nanotube; DW: Distilled water; GNP: Graphene nanoplatelet; ICP-MS: Inductively coupled plasma mass spectrometry;
ND: Nanodiamond; PBS: Phosphate-buffered saline; PK: Proteinase K; PTA: Programmed thermal analysis; SEM: Standard error of the mean; TEM: Transmission electron microscopy

\section{Acknowledgements}

Not applicable.

\section{Authors' contributions}

W.-S.C. and K.S.S. conceived the idea including the development and design of methodology. D.K.L., S.J., and J.J. performed experimentS. D.K.L. and W.-S.C. wrote the manuscript with input from other co-authors. The author(s) read and approved the final manuscript.

\section{Funding}

This research was supported by the BB21+ Project in 2019, the National Research Foundation of Korea (NRF-2019R1A2C1084489), and the Korea Evaluation Institute of Industrial Technology from the Korean Ministry of Trade, Industry \& Energy (20011630).

\section{Availability of data and materials}

The datasets used and/or analyzed during the current study are available from the corresponding author on reasonable request.

Ethics approval and consent to participate

All animal studies obtained proper ethics approval from the Institutional Animal Care and Use Committee at Dong-A University (DIACUC-20-2).

\section{Consent for publication}

Not applicable.

\section{Competing interests}

The authors declare they have no competing interests.

\section{Author details}

${ }^{1}$ Lab of Toxicology, Department of Health Sciences, Dong-A University, 37, Nakdong-daero 550 beon-gil, Saha-gu, Busan 49315, Republic of Korea. ${ }^{2}$ Korea Conformity Laboratories, 8, Gaetbeol-ro 145 beon-gil, Yeonsu-gu, 21999 Incheon, Republic of Korea.

Received: 13 May 2020 Accepted: 2 September 2020

Published online: 11 September 2020

\section{References}

1. Braakhuis HM, Gosens I, Krystek P, Boere JA, Cassee FR, Fokkens PH, Post JA, van Loveren $H$, Park MV. Particle size dependent deposition and pulmonary inflammation after short-term inhalation of silver nanoparticles. Part Fibre Toxicol. 2014;11:49.

2. Braakhuis HM, Park MV, Gosens I, De Jong WH, Cassee FR. Physicochemical characteristics of nanomaterials that affect pulmonary inflammation. Part Fibre Toxicol. 2014;11:18.

3. Sturm R. A stochastic model of carbon nanotube deposition in the airways and alveoli of the human respiratory tract. Inhal Toxicol. 2016;28(2):49-60.

4. Schneider CS, Xu Q, Boylan NJ, Chisholm J, Tang BC, Schuster BS, Henning $A$, Ensign LM, Lee E, Adstamongkonkul $P$, et al. Nanoparticles that do not adhere to mucus provide uniform and long-lasting drug delivery to airways following inhalation. Sci Adv. 2017;3(4):e1601556.

5. Kim JK, Jo MS, Kim Y, Kim TG, Shin JH, Kim BW, Kim HP, Lee HK, Kim HS, Ahn K, et al. 28-day inhalation toxicity study with evaluation of lung deposition and retention of tangled multi-walled carbon nanotubes. Nanotoxicology. 2020;14(2):250-62.

6. Tran CL, Buchanan D, Cullen RT, Searl A, Jones AD, Donaldson K. Inhalation of poorly soluble particles. II. Influence of particle surface area on inflammation and clearance. Inhal Toxicol. 2000;12(12):1113-26.

7. OECD: Test No. 413: Subchronic Inhalation Toxicity: 90-day Study. 2018.

8. OECD: Test No. 412: Subacute Inhalation Toxicity: 28-Day Study. 2018.

9. Doudrick K, Corson N, Oberdorster G, Elder AC, Herckes P, Halden RU, Westerhoff $P$. Extraction and quantification of carbon nanotubes in biological matrices with application to rat lung tissue. ACS Nano. 2013;7(10): 8849-56. 
10. Zhang M, Xu Y, Yang M, Yudasaka M, Okazaki T. Clearance of Single-Wall carbon nanotubes from the mouse lung: a quantitative evaluation. Nanoscale Adv. 2020;2(4):1551-9.

11. Wang R, Mikoryak C, Chen E, Li S, Pantano P, Draper RK. Gel electrophoresis method to measure the concentration of single-walled carbon nanotubes extracted from biological tissue. Anal Chem. 2009;81(8):2944-52.

12. Ingle T, Dervishi E, Biris AR, Mustafa T, Buchanan RA, Biris AS. Raman spectroscopy analysis and mapping the biodistribution of inhaled carbon nanotubes in the lungs and blood of mice. J Appl Toxicol. 2013;33(10):1044-52.

13. Septiadi D, Rodriguez-Lorenzo L, Balog S, Spuch-Calvar M, Spiaggia G, Taladriz-Blanco P, Barosova H, Chortarea S, Clift MJD, Teeguarden J, et al. Quantification of Carbon Nanotube Doses in Adherent Cell Culture Assays Using UV-VIS-NIR Spectroscopy. Nanomaterials. 2019;9(12):1765.

14. IARC. IARC Monographs on the Evaluation of Carcinogenic Risks to Humans. In: Some Nanomaterials and Some Fibres. Lyon: International Agency for Research on Cancer; 2017.

15. Lee JK, Jeong AY, Bae J, Seok JH, Yang JY, Roh HS, Jeong J, Han Y, Jeong J, Cho WS. The role of surface functionalization on the pulmonary inflammogenicity and translocation into mediastinal lymph nodes of graphene nanoplatelets in rats. Arch Toxicol. 2017;91(2):667-76.

16. Nagai H, Okazaki Y, Chew SH, Misawa N, Yamashita Y, Akatsuka S, Ishihara T, Yamashita K, Yoshikawa Y, Yasui H. Diameter and rigidity of multiwalled carbon nanotubes are critical factors in mesothelial injury and carcinogenesis. Proc Natl Acad Sci U S A. 2011;108(49):E1330-8.

17. Muhlfeld C, Poland CA, Duffin R, Brandenberger C, Murphy FA, RothenRutishauser B, Gehr P, Donaldson K. Differential effects of long and short carbon nanotubes on the gas-exchange region of the mouse lung. Nanotoxicology. 2012;6:867-79

18. Rasmussen K, Rauscher H, Kearns P, González M, Riego Sintes J. Developing OECD test guidelines for regulatory testing of nanomaterials to ensure mutual acceptance of test data. Regul Toxicol Pharmacol. 2019;104:74-83.

19. Worsley KA, Kalinina I, Bekyarova E, Haddon RC. Functionalization and dissolution of nitric acid treated single-walled carbon nanotubes. J Am Chem Soc. 2009;131(50):18153-8.

20. Gosens I, Cassee FR, Zanella M, Manodori L, Brunelli A, Costa AL, Bokkers BG, de Jong WH, Brown D, Hristozov D, et al. Organ burden and pulmonary toxicity of nano-sized copper (II) oxide particles after short-term inhalation exposure. Nanotoxicology. 2016;10(8):1084-95.

21. Jo MS, Kim JK, Kim Y, Kim HP, Kim HS, Ahn K, Lee JH, Faustman EM, Gulumian M, Kelman B, et al. Mode of silver clearance following 28-day inhalation exposure to silver nanoparticles determined from lung burden assessment including post-exposure observation periods. Arch Toxicol. 2020; 94(3):773-84.

22. Morton J, Tan E, Suvarna SK. Multi-elemental analysis of human lung samples using inductively coupled plasma mass spectrometry. J Trace Elem Med Biol. 2017;43:63-71.

23. Cho WS, Duffin R, Howie SE, Scotton CJ, Wallace WA, Macnee W, Bradley M, Megson IL, Donaldson K. Progressive severe lung injury by zinc oxide nanoparticles; the role of $\mathrm{Zn} 2+$ dissolution inside lysosomes. Part Fibre Toxicol. 2011;8:27.

24. Li R, Ji Z, Chang CH, Dunphy DR, Cai X, Meng H, Zhang H, Sun B, Wang X, Dong J, et al. Surface interactions with compartmentalized cellular phosphates explain rare earth oxide nanoparticle hazard and provide opportunities for safer design. ACS Nano. 2014;8(2):1771-83.

25. Ohnishi M, Suzuki M, Yamamoto M, Kasai T, Kano H, Senoh H, Higashikubo I, Araki A, Fukushima S, et al. Improved method for measurement of multiwalled carbon nanotubes in rat lung. J Occup Med Toxicol. 2016;11:44

26. Birch ME. Occupational monitoring of particulate diesel exhaust by NIOSH method 5040. Appl Occup Environ Hyg. 2002;17(6):400-5.

27. Elgrabli D, Floriani M, Abella-Gallart S, Meunier L, Gamez C, Delalain P, Rogerieux F, Boczkowski J, Lacroix G. Biodistribution and clearance of instilled carbon nanotubes in rat lung. Particle Fibre Toxicol. 2008;5(1):20.

28. Rance GA, Marsh DH, Nicholas RJ, Khlobystov AN. UV-vis absorption spectroscopy of carbon nanotubes: relationship between the $\pi$-electron plasmon and nanotube diameter. Chem Phys Lett. 2010;493(1):19-23.

29. Fagan JA, Landi BJ, Mandelbaum I, Simpson JR, Bajpai V, Bauer BJ, Migler K, Hight Walker AR, Raffaelle R, Hobbie K. Comparative measures of Single-Wall carbon nanotube dispersion. J Phys Chem B. 2006;110(47):23801-5.

30. Yang B, Ren L, Li L, Tao X, Shi Y, Zheng Y. The characterization of the concentration of the single-walled carbon nanotubes in aqueous dispersion by UV-Vis-NIR absorption spectroscopy. Analyst. 2013;138(21):6671-6.
31. Lee DK, Jeon S, Han Y, Kim SH, Lee S, Yu IJ, Song KS, Kang A, Yun WS, Kang SM, et al. Threshold rigidity values for the Asbestos-like pathogenicity of high-aspect-ratio carbon nanotubes in a mouse pleural inflammation model. ACS Nano. 2018;12(11):10867-79.

32. Bihari $P$, Vippola $M$, Schultes $S$, Praetner $M$, Khandoga AG, Reichel CA, Coester C, Tuomi T, Rehberg M, Krombach F. Optimized dispersion of nanoparticles for biological in vitro and in vivo studies. Part Fibre Toxicol. 2008:5:14.

33. Murphy FA, Poland CA, Duffin R, Al-Jamal KT, Ali-Boucetta $H$, Nunes A, Byrne F, Prina-Mello A, Volkov Y, Li S, et al. Length-dependent retention of carbon nanotubes in the pleural space of mice initiates sustained inflammation and progressive fibrosis on the parietal pleura. Am J Pathol. 2011;178(6):2587600.

34. Shinohara N, Nakazato T, Ohkawa K, Tamura M, Kobayashi N, Morimoto Y, Oyabu T, Myojo T, Shimada M, Yamamoto K, et al. Long-term retention of pristine multi-walled carbon nanotubes in rat lungs after intratracheal instillation. J Appl Toxicol. 2016;36(4):501-9.

35. Xiang L, Yuan Y, Da X, Ou Z, Yang S, Zhou F. Photoacoustic molecular imaging with antibody-functionalized single-walled carbon nanotubes for early diagnosis of tumor. J Biomed Opt. 2009;14(2):021008.

36. Gao S, Qiu H, Zhang C, Jiang S, Li Z, Liu X, Yue W, Yang C, Huo Y, Feng D. Absorbance response of a graphene oxide coated U-bent optical fiber sensor for aqueous ethanol detection. RSC Adv. 2016;6(19):15808-15.

37. Bond TC, Bergstrom RW. Light absorption by carbonaceous particles: an investigative review. Aerosol Sci Technol. 2006;40(1):27-67.

\section{Publisher's Note}

Springer Nature remains neutral with regard to jurisdictional claims in published maps and institutional affiliations.
Ready to submit your research? Choose BMC and benefit from:

- fast, convenient online submission

- thorough peer review by experienced researchers in your field

- rapid publication on acceptance

- support for research data, including large and complex data types

- gold Open Access which fosters wider collaboration and increased citations

- maximum visibility for your research: over $100 \mathrm{M}$ website views per year

At $\mathrm{BMC}$, research is always in progress.

Learn more biomedcentral.com/submissions 